PROTOCOL OPEN

\title{
Systematic scoping review protocol of methodologies of chronic respiratory disease surveys in low/middle-income
} countries

Dhiraj Agarwal (iD) ${ }^{1}$, Nik Sherina Hanafi (iD ${ }^{2}$, Soumya Chippagiri ${ }^{3}$, Evelyn A. Brakema (iD) ${ }^{4}$, Hilary Pinnock ${ }^{5}$, Ee Ming Khoo (iD ${ }^{2}$, Aziz Sheikh ${ }^{5}$, Su-May Liew ${ }^{2}$, Chiu-Wan $\mathrm{Ng}^{2}$, Rita Isaac ${ }^{3}$, Karuthan Chinna ${ }^{2}$, Wong Li Ping $\mathbb{D}^{2}$, Norita Binti Hussein ${ }^{2}$, Sanjay Juvekar (iD) and the RESPIRE Collaborators

This protocol describes a systematic scoping review of chronic respiratory disease surveys in low/middle-income countries (LMICs) undertaken as part of the Four Country ChrOnic Respiratory Disease (4CCORD) study within the National Institute for Health Research Global Health Research Unit on Respiratory Health (RESPIRE). Understanding the prevalence and burden of chronic respiratory disease (CRD) underpins healthcare planning. We will systematically scope the literature to identify existing strategies (definitions/questionnaires/diagnostics/outcomes) used in surveys of CRDs in adults in low-resource settings. We will search MEDLINE, EMBASE, ISI WoS, Global Health and WHO Global Health Library [search terms: prevalence AND CRD (COPD, asthma) AND LMICs, from 1995], and two reviewers will independently extract data from selected studies onto a piloted customised data extraction form. We will convene a workshop of the multidisciplinary 4CCORD research team with representatives from the RESPIRE partners (Bangladesh, India, Malaysia, Pakistan and Edinburgh) at which the findings of the scoping review will be presented, discussed and interpreted. The findings will inform a future RESPIRE 4CCORD study, which will estimate CRD burden in adults in Asian LMICs.

npj Primary Care Respiratory Medicine (2019)29:17; https://doi.org/10.1038/s41533-019-0129-7

\section{BACKGROUND}

Chronic respiratory diseases (CRDs), especially asthma and chronic obstructive pulmonary disease (COPD), are common public health problems across the world, with the Global Burden of Disease estimating that CRDs now account for 30\% of total deaths. ${ }^{1}$ Although morbidity and mortality are particularly high in low- and middle-income countries (LMICs), there are very little robust data on the true prevalence of asthma and COPD in these countries. ${ }^{2,3}$ Chronic respiratory symptoms are common in the general population, ${ }^{1}$ but the clinicians in primary healthcare systems in resource-poor countries lack the skills and support to diagnose the underlying disease condition. ${ }^{4-8}$ Factors contributing to low rates of diagnosis include limited awareness of respiratory long-term conditions, ${ }^{4-10}$ limited access to healthcare and lack of diagnostic capability in these countries. ${ }^{11}$ Determining the prevalence of asthma and COPD remains a challenge because of the poor sensitivity and specificity of the widely used questionnaire-based research tools, ${ }^{3,12}$ while objective testing with spirometry may be a challenge in community-based epidemiological surveys. ${ }^{11}$

With notable exceptions, such as the Burden of Lung Disease (BOLD), ${ }^{13}$ surveys of the prevalence of CRDs conducted in LMICs often have major limitations (such as relying on patient-reported disease or symptom questionnaires), and report very varied estimates of prevalence. ${ }^{2,14-39}$ In addition, many existing surveys focus on one condition (e.g. the BOLD study detects COPD, ${ }^{13}$ the International Study of Asthma and Allergies in Childhood (ISAAC) detects symptoms of asthma and allergy in children ${ }^{40}$ ) and rarely look for the broad range of less common causes of CRD (such as interstitial lung disease, bronchiectasis, lung cancer and complications post tuberculosis) or attempt to identify the phenotypes of asthma and COPD, which are increasingly regarded as important to understanding and managing the conditions. ${ }^{41}$

Effective health policies related to CRD can only be developed if we know the true burden of asthma, COPD and other CRDs in the community. Funded by the National Institute for Health Research (NIHR), RESPIRE is a Global Health Research Unit focusing on respiratory health in Asia (https://www.ed.ac.uk/usher/respire). Prior to undertaking a comprehensive Four Country ChrOnic Respiratory Disease study (4CCORD) in the partner countries of RESPIRE, we sought to systematically scope the literature to identify existing strategies-that is, definitions, questionnaires, study tools and diagnostics protocols-that have been used to conduct surveys for CRDs in LMICs.

\footnotetext{
'Vadu Rural Health Program, KEM Hospital Research Centre, Pune, India; ${ }^{2}$ Department of Primary Care Medicine, Faculty of Medicine, University of Malaya, Kuala Lumpur, Malaysia; ${ }^{3}$ RUHSA Department, Christian Medical College, Vellore, India; ${ }^{4}$ Department of Public Health and Primary care, Leiden University Medical Centre, Leiden, The Netherlands and ${ }^{5} \mathrm{NIHR}$ Global Health Research Unit on Respiratory Health (RESPIRE), Usher Institute of Population Health Sciences and Informatics, The University of Edinburgh, Edinburgh, UK

Correspondence: Sanjay Juvekar (sanjay.juvekar@gmail.com)

A list of consortium members appears before the Acknowledgements.

These authors contributed equally: Dhiraj Agarwal, Nik Sherina Hanafi
}

Received: 3 March 2019 Accepted: 12 April 2019

Published online: 08 May 2019 
Table 1. Inclusion and exclusion criteria

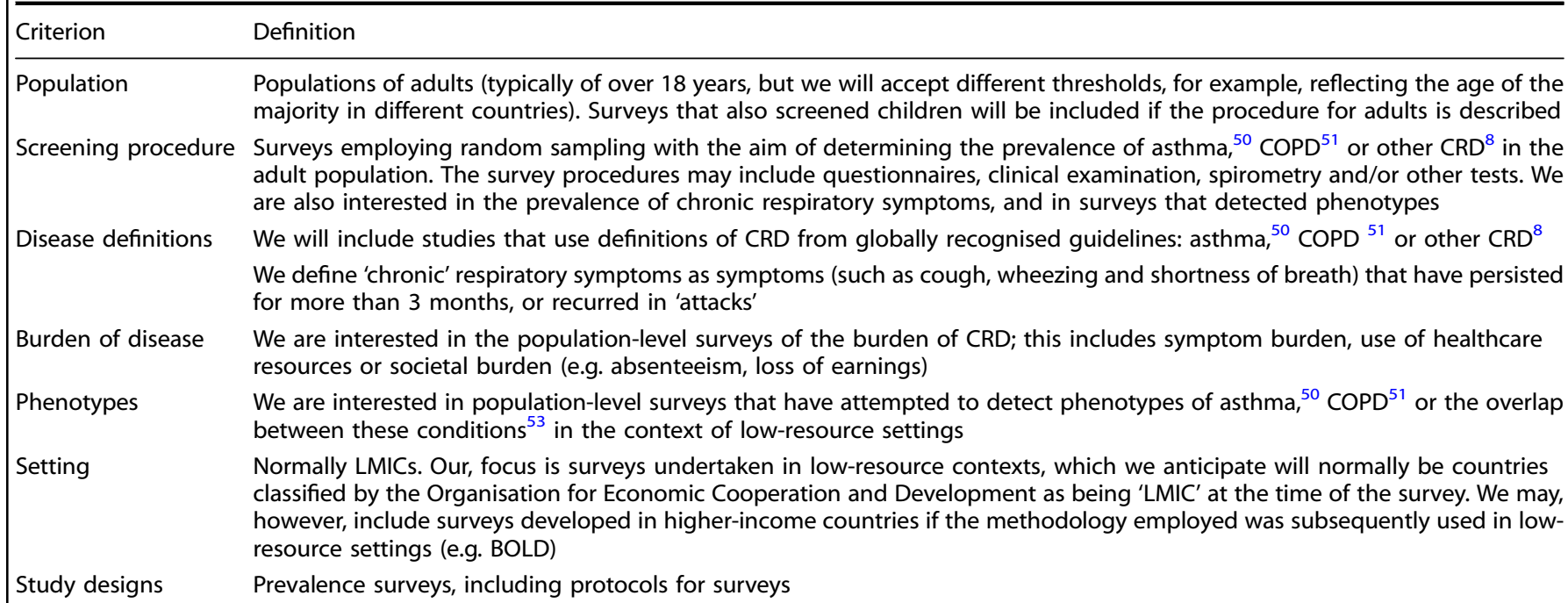

COPD chronic obstructive pulmonary disease, CRD chronic respiratory disease, LMICs low- and middle-income countries, BOLD Burden of Lung Disease

\section{METHODS}

A systematic scoping review aims to 'map the key concepts underpinning a research area and the main sources and types of evidence available'. ${ }^{42}$ As such, scoping reviews typically address broad questions, potentially include a range of methodologies and do not undertake quality assessment. This contrasts with the focused question of a systematic review, which is answered from a relatively narrow range of qualityassessed studies. ${ }^{42}$

Systematic review procedures, as described in the Cochrane Handbook for Systematic Reviews of Interventions, ${ }^{43}$ have been adapted to meet the needs of a scoping review. ${ }^{44,45}$ We will follow the six-step framework described by Arksey and O'Malley ${ }^{42}$ and subsequently widely endorsed and enhanced in methodological literature. ${ }^{45-47}$

\section{Identifying the research question}

The aim of this review is to scope published epidemiological CRD surveys to identify their aims, methodologies and outcomes in order to identify evidence gaps and inform the methodology of the proposed RESPIRE 4CCORD survey on the burden of CRD. Our objectives are therefore to answer the following research questions:

1. What surveys on the prevalence of asthma, COPD and other CRDs have been undertaken in resource-poor settings?

2. What definitions, questionnaires, tests, diagnostic processes and outcomes did the surveys employ?

3. How was the socio-economic burden (from a societal or healthcare perspective) of asthma, COPD and other CRDs estimated in these surveys?

4. What strategies have been used to identify phenotypes of asthma and COPD, or to identify the causes of 'other CRD'?

\section{Identifying relevant studies}

We will search MEDLINE, EMBASE, ISI Web of Science, Global Health and WHO Global Health Library using search terms for prevalence AND CRD (COPD OR asthma OR CRD) AND LMICs/low-resource settings, from year 1995 onwards when publication of the Global Initiative for Asthma (GINA) ${ }^{48}$ followed by the Global Initiative for Chronic Obstructive Lung Disease (GOLD) guidelines for COPD, ${ }^{49}$ provided internationally agreed definitions and diagnostic strategies for the two most common CRDs. The detailed MEDLINE search strategy is shown in Supplementary Table 1.

Key websites (e.g. http://ghdx.healthdata.org) will be checked for eligible studies. We will follow up references of surveys with methodological papers, and will group papers from the same study (e.g. BOLD, ${ }^{13}$ FRESH $\mathrm{AlR}^{4}$ ). To avoid overlooking relevant local studies, we will not impose language restrictions; translations will be undertaken where possible.

\section{Study selection}

Selection criteria and definitions are detailed in Table 1. Our focus is on surveys conducted in LMICs to study the prevalence of CRD (specifically asthma and COPD but also other CRDs) in randomly sampled communitybased populations of adults. We anticipate that these will incorporate assessment of chronic respiratory symptoms and objective tests, measurement of risk factors for CRD, phenotypes of asthma/COPD or individual, healthcare or societal burden of CRD. We will undertake training at each step (100 randomly selected titles/abstracts or 50 full-text papers will be screened independently by D.A., N.S.H., H.P., E.M.K. and S.J.; they will be repeated until agreement exceeds $90 \%$ ). After an initial shift to exclude obviously irrelevant titles (D.A., N.S.H.), two reviewers (D.A., N.S.H., S.C., S.-M.L. or E.A.B.) will screen the titles and abstracts, and then undertake full-text screening of potentially relevant papers, with disagreements resolved by discussion between researchers, arbitrated by a third reviewer (H.P., E.M.K. and S.J.) if necessary, and involving the wider research group to agree on conventions that operationalise the inclusion/exclusion criteria (see Table 1). The selection process will be undertaken using EndNote software (v9.0) and summarised using a PRISMA flow diagram.

\section{Charting the data}

Two reviewers (D.A., N.S.H., S.C., S.-M.L. or E.A.B.) will independently extract data onto a piloted customised data extraction form as follows: study metadata, country and populations, sampling strategy, CRD definitions, survey procedure (questionnaires used, spirometry and other measurements), risk factors measured, assessments of individual, societal and health service burden of disease and detection of phenotypes. Our research questions focus on identifying the process (rather than the outcomes) of undertaking surveys of CRD in LMICs, though we will note the prevalence of asthma, COPD and any other CRD identified in the included studies in order to inform future sample size calculations.

We will attempt to contact authors of the included papers for missing or unclear essential information; specifically, we will request copies of questionnaires or study procedures if they are not otherwise available. Multiple publications from the same study will be grouped, and draw on all the relevant publications, including methodological papers. ${ }^{13}$

Collating, summarising and reporting the results We will tabulate the procedures used and identify

- The strategies used to identify randomly sampled populations in LMICs

- The disease definitions used (which may vary over time)

- The questionnaires used and tests performed to detect asthma, COPD and/or other CRD 
- Other variables for which information is collected in the included studies, in order to assess individual, societal and healthcare burden of CRD, and the risk factors for disease

- Whether recent surveys have addressed contemporary understanding of asthma/COPD phenotypes ${ }^{41,50,51}$ and the variables assessed to achieve this in low-resource settings

\section{Consultation}

We will convene a workshop of the multidisciplinary research team with representatives from the RESPIRE partners (from Bangladesh, India, Malaysia, Pakistan and Edinburgh), at which the findings of the scoping review will be presented, discussed and interpreted. The conclusions will inform a future RESPIRE 4CCORD study, which will aim to estimate CRD burden in adults in Asian LMICs (initially Bangladesh, India, Malaysia and Pakistan).

\section{Dissemination}

We will publish our findings in a peer-reviewed journal following the reporting standards for scoping reviews (PRISMA-SCR) ${ }^{52}$ and disseminate widely through our stakeholder groups and using innovative media approaches.

\section{DISCUSSION}

Assessment of the true prevalence for asthma, COPD and other CRDs underpins healthcare planning. Policymakers need assessments of the burden of disease if they are to plan and resource services that can accurately diagnose and effectively reduce the burden of CRD. Communication of the burden of CRD will raise awareness amongst stakeholders of the individual and societal impact of respiratory conditions and highlight the importance to communities of addressing potentially modifiable risk factors.

Limitations in currently available estimates of CRD burden in South Asian countries (e.g. relying on symptom questionnaires, or patient-reported diagnoses), hamper advocacy and are a barrier to development of healthcare service. Our scoping review will identify existing strategies used in surveys in LMICs, enabling future surveys to optimise their methodology and provide robust estimates of disease burden.

\section{THE RESPIRE COLLABORATORS}

D. Das ${ }^{3}$, B. Paul ${ }^{3}$, H. Campbell, E. Grant ${ }^{5}$, M. Fletcher ${ }^{5}$, S. Saha ${ }^{6}$, M. Habib ${ }^{7}$, S. El

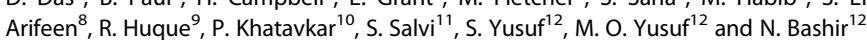

${ }^{6}$ Child Health Research Foundation, Dhaka, Bangladesh; ${ }^{7}$ Bangladesh Primary Care Respiratory Society, Dhaka, Bangladesh; ${ }^{8}$ Maternal and Child Health Division (MCHD), International Centre for Diarrhoeal Disease Research, Dhaka, Bangladesh; ${ }^{9}$ University of Dhaka, Dhaka, Bangladesh; ${ }^{10} \mathrm{KEM}$ Hospital, Pune, India; ${ }^{11}$ Chest Research Foundation, Pune, India and ${ }^{12}$ The Allergy \& Asthma Institute, Islamabad, Pakistan

\section{ACKNOWLEDGEMENTS}

We gratefully acknowledge the advice from Dr. Marshall Dozier, Academic Support Librarian, University of Edinburgh for her help with developing the search strategy. We also acknowledge Shalini Selvaratnam, the University of Malaya, who helped with screening papers and data extraction. We are also grateful to the peer reviewers whose comments helped us refine our proposal and the RESPIRE collaborators. This scoping review is one of the projects within the pilot phase of the 4CCORD study, which is funded by the NIHR Global Health Research Unit in Respiratory Health (RESPIRE) at the Usher Institute of Population Health Sciences and Informatics. RESPIRE was commissioned by the National Institute of Health Research using Official Development Assistance (ODA) funding. The views expressed are those of the ?author(s) and not necessarily those of the NHS, the NIHR or the Department of Health and Social Care.

\section{AUTHOR CONTRIBUTIONS}

S.J., E.M.K. and H.P. led the development of the scoping review with D.A. and N.S.H., and with the advice of other members of the 4CCORD group. D.A. wrote the first draft of the protocol, which was critically reviewed and refined by S.J., E.M.K., H.P., N.S.H., S. C., S.-M.L., E.A.B. and A.S.A. RESPIRE UMC members and Co-Is of the 4CCORD study provided advice and contributed to discussions from time to time. All authors approved the final version. D.A. and N.S.H. contributed equally to the development, conduct, interpretation of findings and writing of the paper.

\section{ADDITIONAL INFORMATION}

Supplementary information accompanies the paper on the npj Primary Care Respiratory Medicine website (https://doi.org/10.1038/s41533-019-0129-7).

Competing interests: The authors declare no competing interests.

Publisher's note: Springer Nature remains neutral with regard to jurisdictional claims in published maps and institutional affiliations.

\section{REFERENCES}

1. Mathers, C. D. \& Loncar, D. Projections of global mortality and burden of disease from 2002 to 2030. PLoS Med. 3, e442 (2006).

2. Adeloye, D. et al. Global and regional estimates of COPD prevalence: systematic review and meta-analysis. J. Global Health 5, 1-7 (2015).

3. Salvi, S. S., Manap, R. \& Beasley, R. Understanding the true burden of COPD: the epidemiological challenges. Prim. Care Respir. J. 21, 249-251 (2012).

4. Van Gemert, F. et al. Prevalence of chronic obstructive pulmonary disease and associated risk factors in Uganda (FRESH AIR Uganda): a prospective crosssectional observational study. Lancet Global Health 3, e44-e51 (2015).

5. WHO. Monitoring the Building Blocks of Health Systems: A Handbook of Indicators and their Measurement Strategies 1-92 (WHO, Geneva, 2010).

6. Banda, H. T. et al. Community prevalence of chronic respiratory symptoms in rural Malawi: implications for policy. PLOS ONE 1623, 1-13 (2017).

7. Lainez, Y. B., Todd, C. S., Ahmadzai, A., Doocy, S. C. \& Burnham, G. Prevalence of respiratory symptoms and cases suspicious for tuberculosis among public health clinic patients in Afghanistan, 2005-2006: perspectives on recognition and referral of tuberculosis cases. Trop. Med. Int. Health 14, 564-570 (2009).

8. Bousquet, J., Dahl, R. \& Khaltaev, N. GARD (Global Alliance against chronic Respiratory Diseases). Rev. Mal. Respir. 23, 73-75 (2006).

9. Østergaard, M. S. et al. Recurrent lower respiratory illnesses among young children in rural Kyrgyzstan: overuse of antibiotics and possible underdiagnosis of asthma. A qualitative FRESH AIR study. npj Prim. Care Respir. Med. 28, 13 (2018).

10. Salvi, S. et al. Symptoms and medical conditions in 204912 patients visiting primary health-care practitioners in India: a 1-day point prevalence study (the POSEIDON study). Lancet Global Health 3, e776-e784 (2015).

11. Bousquet, J., Dahl, R. \& Khaltaev, N. Global Alliance against Chronic Respiratory Diseases. Eur. J. Allergy Clin. Immunol. 62, 216-223 (2007).

12. Contoli, M. \& Papi, A. When asthma diagnosis becomes a challenge. Eur. Respir. J. 36, 231-233 (2010)

13. Buist, A. S. et al. The Burden of Obstructive Lung Disease Initiative (BOLD): rationale and design. J. Chronic Obstr. Pulm. Dis. 2, 277-283 (2005).

14. Anandan, C., Nurmatov, U., Van Schayck, O. C. P. \& Sheikh, A. Is the prevalence of asthma declining? Systematic review of epidemiological studies. Eur. J. Allergy Clin. Immunol. 65, 152-167 (2010).

15. Salvi, S. \& Barnes, P. J. Is exposure to biomass smoke the biggest risk factor for COPD globally? Chest J. 138, 3-6 (2010).

16. Nasir, K. R. N. Epidemiology of cigarette smoking in Pakistan. Addiction 96 1847-1854 (2001).

17. Nargis, N. et al. Prevalence and patterns of tobacco use in Bangladesh from 2009 to 2012: evidence from International Tobacco Control (ITC) study. PLoS ONE 10, 1-16 (2015).

18. Lim, H. K. et al. Epidemiology of smoking among Malaysian adult males: prevalence and associated factors. BMC Public Health 13, 1 (2013).

19. Mishra, S. et al. Trends in bidi and cigarette smoking in India from 1998 to 2015, by age, gender and education. BMJ Global Health 1, 1-8 (2016).

20. Lim, S. et al. Impact of chronic obstructive pulmonary disease (COPD) in the AsiaPacific region: the EPIC Asia population-based survey. Asia Pac. Fam. Med. 14, 4 (2015).

21. Ahmed, N. Study on Prevalence of Asthma and COPD at Dhaka City in Bangladesh. Dissertation, East West Univ., Dhaka (2016).

22. Alam, D. S., Chowdhury, M. A., Siddiquee, A. T., Ahmed, S. \& Clemens, J. D. Prevalence and determinants of chronic obstructive pulmonary disease (COPD) in Bangladesh. COPD J. Chronic Obstr. Pulm. Dis. 12, 658-667 (2015).

23. WHO. Global Tuberculosis Report 2017. Licence: CC BY-NC-SA 3.0 IGO (World Health Organization, Geneva, 2017). 
24. Sultana, T., Afzal, A., Sultana, S., Al-ghanim, K. \& Shahid, T. Epidemiological estimates of respiratory diseases in the hospital population, Faisalabad, Pakistan. Braz. Arch. Biol. Technol. 60, 1-12 (2017).

25. Subbarao, P. Asthma: epidemiology, etiology and risk factors. Can. Med. Assoc. J. 181, E181-E190 (2009).

26. Salvi, S. \& Agrawal, A. India needs a National COPD Prevention and Control Programme. J. Assoc. Physicians India 60, 5-7 (2012).

27. Zhang, Li. Lung cancer in China: challenges and perspectives. J. Thorac. Oncol. 12, S113-S114 (2017).

28. Singh, V. \& Sharma, B. B. The ILD India Registry: a novel tool for epidemiological surveillance of interstitial lung disease in India. Indian J. Chest Dis. Allied Sci. 55, 197-199 (2013).

29. Jahid, H. Interstitial lung disease (ILD) in India: Insights and lessons from the prospective, landmark ILD-India registry. Lung India 33, 589-591 (2016).

30. Dhar, R. Bronchiectasis in India: More Questions than Answers. RespiMirror (Chest Research Foundation, Pune, 2015).

31. Chalmers, J. D. et al. The European Multicentre Bronchiectasis Audit and Research Collaboration (EMBARC): experiences from a successful ERS clinical research collaboration. Breathe 13, 180-192 (2017).

32. Mandal, A., Kabra, S. K. \& Lodha, R. Cystic fibrosis in India: past, present and future. J. Pulm. Med. Respir. Res. 1, 1-8 (2015)

33. Jindal, S. K. Indian Study on Epidemiology of Asthma, Respiratory Symptoms and Chronic Bronchitis (INSEARCH). Rep. Indian Counc. Med. Res. 335, 1270-1277 (2010).

34. Bishwajit, G., Tang, S., Yaya, S. \& Feng, Z. Burden of asthma, dyspnea, and chronic cough in South Asia. Int. J. COPD 12, 1093-1099 (2017).

35. De Santi, F. et al. Type 2 diabetes is associated with an increased prevalence of respiratory symptoms as compared to the general population. BMC Pulm. Med. 17, 101 (2017).

36. Bartlett, E., Parr, J., Lindeboom, W., Khanam, A. \& Koehlmoos, T. P. Sources and prevalence of self- reported asthma diagnoses in adults in urban and rural settings of Bangladesh. Global Public Health 8, 37-41 (2012).

37. Ahad, A. \& Ming Khoo, E. Asthma control and care among Malaysian primary school children: a cross-sectional study. Asia Pac. J. Public Health 29, 422-429 (2017).

38. Chan, Y. Y. et al. Lifestyle, chronic diseases and self-rated health among Malaysian adults: results from the 2011 National Health and Morbidity Survey (NHMS). BMC Public Health 15, 1-12 (2015).

39. Loh, L. C. et al. Low prevalence of obstructive lung disease in a suburban population of Malaysia: a BOLD collaborative study. Respirology 21, 1055-1061 (2016).

40. Lai, C. K. W. et al. Global variation in the prevalence and severity of asthma symptoms: phase three of the International Study of Asthma and Allergies in Childhood (ISAAC). Thorax 64, 476-483 (2009).

41. Pavord, I. D. et al. After asthma-redefining airways diseases. A Lancet commission. Lancet 391, 350-400 (2017).

42. Arksey, H. \& O'Malley, L. Scoping studies: towards a methodological framework. Int. J. Soc. Res. Methodol. 8, 19-32 (2005).
43. Chandler, J., Higgins, J. P. T., Deeks, J. J., Davenport, C. \& Clarke, M. J. in Cochrane Handbook for Systematic Reviews of Interventions Version 5.2.0 (eds Higgins, J. P. T., Churchill, R., Chandler, J. \& Cumpston, M. S.) (Cochrane, https://community. cochrane.org/handbook-sri 2017). Available from Cochrane Community.

44. Armstrong, R., Hall, B. J., Doyle, J. \& Waters, E. "Scoping the scope" of a Cochrane review. J. Public Health (Bangk.). 33, 147-150 (2011).

45. Peters, M. D. J. et al. Guidance for conducting systematic scoping reviews. Int. J. Evid. Based Healthc. 13, 141-146 (2015).

46. Levac, D., Colquhoun, H. \& O'Brien, K. K. Scoping studies: advancing the methodology. Implement. Sci. 5, 1-9 (2010).

47. Colquhoun, H. L. et al. Scoping reviews: time for clarity in definition, methods, and reporting. J. Clin. Epidemiol. 67, 1291-1294 (2014).

48. Bateman, E. D. et al. Global strategy for asthma management and prevention: GINA executive summary. Eur. Respir. J. 31, 143-178 (2008).

49. Pauwels, R. A., Buist, A. S., Calverley, P. M. A., Jenkins, C. R. \& Hurd, S. S. NHLBI/ WHO Workshop Summary. Global Strategy for the Diagnosis, Management, and Prevention of Chronic Obstructive Pulmonary Disease NHLBI/WHO Global Initiative for Chronic Obstructive Lung Disease (GOLD) Workshop Summary. Am. J. Respir. Crit. Care Med. 163, 1256-1276 (2001).

50. Global Initiative for Asthma. Global Strategy for Asthma Management and Prevention. www.ginasthma.org (2017).

51. Global Initiative for Chronic Obstructive Lung Disease. Global Strategy for the Diagnosis, Management, and Prevention of Chronic Obstructive Pulmonary Disease Report. (2017).

52. Tricco, A. C. et al. PRISMA Extension for Scoping Reviews (PRISMA-ScR): checklist and explanation. Ann. Intern. Med. 169, 467-473 (2018).

53. Global Initiative for Asthma and Global Initiative for Chronic Obstructive Lung Disease Diagnosis of Diseases of Chronic Airflow Limitations: Asthma, COPD and Asthma-COPD Overlap Syndrome (ACOS). (2015).

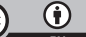

Open Access This article is licensed under a Creative Commons Attribution 4.0 International License, which permits use, sharing, adaptation, distribution and reproduction in any medium or format, as long as you give appropriate credit to the original author(s) and the source, provide a link to the Creative Commons license, and indicate if changes were made. The images or other third party material in this article are included in the article's Creative Commons license, unless indicated otherwise in a credit line to the material. If material is not included in the article's Creative Commons license and your intended use is not permitted by statutory regulation or exceeds the permitted use, you will need to obtain permission directly from the copyright holder. To view a copy of this license, visit http://creativecommons. org/licenses/by/4.0/.

(c) The Author(s) 2019 